\title{
What Was the Syntax-Semantics Debate in the Philosophy of Science About?
}

\author{
Sebastian Lutz \\ Preprint: $2015-02-26$
}

\begin{abstract}
The debate between critics of syntactic and semantic approaches to the formalization of scientific theories has been going on for over 40 years. I review the debate in light of a recent exchange between Hans Halvorson, Clark Glymour, and Bas van Fraassen and argue that the only remaining disagreement concerns the alleged difference in the language dependence of syntactic and semantic approaches. This difference turns out to be illusory.

Keywords: semantic approach, Suppes predicate, constructive empiricism, syntactic approach, received view, logical empiricism, model theory, definitional equivalence
\end{abstract}

\section{Introduction}

The basic controversy about syntactic and semantic formalizations of theories seems simple: In syntactic approaches theories are described by sets of sentences, in semantic approaches they are described by classes of model-theoretic structures, and the question is which is preferable. But the debate has been going on since at least the 1960s and has spanned such diverse topics as the problems of formalization in first order logic, the need for formalization in general, the role of models in the sciences, the dependence of scientific theories on their formulation, and the right way of identifying phenomena. It has recently come to a head, with Suppe (2000), Suppes (2002) and Muller (2010) criticizing syntactic approaches, Halvorson (2013) and Lutz (2014d) defending them, Halvorson (2012) criticizing semantic approaches, and Glymour (2013) and van Fraassen (2014) defending them. By the standards of the debate, this most recent exchange has a comparably narrow focus: The question is whether either of the two kinds

* Munich Center for Mathematical Philosophy, Ludwig-Maximilians-Universität München. sebastian.lutz@gmx.net. I thank Holger Andreas, Richard Dawid, Adam Caulton, Samuel Fletcher, Hans Halvorson, Thomas Pashby, and Karim Thébault for helpful discussions. Research for this article has been supported by the Alexander von Humboldt Foundation. 
of approach is in principle unable to describe specific scientific theories. However, discussing only this most recent exchange is well-nigh impossible without taking the history of the debate into account because the recent exchange still contains so many rudiments of previous ones. Even when focused on the central participants and further streamlined, the debate is somewhat unstructured. It goes a bit like this: ${ }^{1}$

Carnap (1939, 1956): I think that we need to get some precision into the discussion about scientific theories. I suggest formalizing theories-and their connections to experiments and observations-using sentences in higher order logic, with one set of terms distinguished as observational and interpreted directly.

P. Suppes $(1967,2002)$ : Well, one should certainly not formalize theories in first order logic. Doing so would often be incredibly difficult and cumbersome. It would be better to define the models of the theory directly using a set theoretic predicate.

Van Fraassen (1970): Or one could describe theories as restrictions on their state-spaces. These state-spaces could then be used to interpret simple elementary sentences like 'quantity $m$ has value $r$ at time $t$ ' that can connect to observations in some more or less difficult way.

F. Suppe (1974b): I think Carnap's suggestion cannot work because it leads to distinctions without a difference: The same theory can be described by two different sets of sentences. By contrast, Suppes predicates and state-space restrictions stay the same. So we should use either of those for formalizing theories.

Van Fraassen (1980, 1989): You know what? We don't need elementary sentences. We can be wholly independent of the language of predicate logic by just sticking with state-space descriptions. We can describe observational data by structures, theories by classes of structures, and the relation between data and theory by the embedding relation. Of course we still need some language to describe all these structures, but at least it does not have to be predicate logic.

F. Suppe (2000): That's good, because most theories cannot even be described syntactically: The Löwenheim-Skolem theorem entails that sentences cannot describe the models of a theory as precisely as is often necessary.

Halvorson (2012): Wait a minute. It seems to me that structures are no good at all for describing theories. Ignoring the relation of a theory to the world for now, I can come up with examples in which the same theory is described

1. I thank Karim Thébault for the suggestion of reproducing the debate as a conversation. 
by different classes of structures, and examples in which two different theories have the same class of structures.

Glymour (2013): I don't think you need to ignore the relation of a theory to the world. Since embedding, for instance, is a purely formal relation, your examples still work. But they fail to work once you take into account that in model theory, structures already come with a language of predicate logic. So one has to take language into account.

Halvorson (2013): But if one uses a language of predicate logic, then there is nothing to the much touted language independence of structures. In fact, then things look downright syntactic to me.

Van Fraassen (2014): I think you are missing the point. I always said that we need some language to describe structures, and my state-space account included elementary sentences anyway. As to the counterexamples: Since they ignore the relation of a theory to the world, they cannot work as a criticism of accounts that include the relation to the world.

In the following, I aim at showing that the above dialogue is an accurate summary of the historical debate and that it contains a number of weak arguments. I will further argue that the exact point of disagreement as it emerges from the debate is so minor that the debate seems to have been about barely anything at all.

\section{The Received View}

One recurring problem of the debate is that it mixes criticisms of syntactic approaches in general with criticisms of the logical empiricists' position, and the recent exchange is no exception. Hence in this section, I will give a short overview of the logical empiricists' position on the formalization of theories. As it turns out, this is already enough to counter some criticisms.

From the 1920 s to the 1960 , the logical empiricists, especially Carnap and Hempel, developed their formal approach to the analysis of scientific theories that Putnam (1962, 240) would later call the "Received View". It is the mostdeveloped and best known syntactic approach. In the Received View, a scientific theory is formalized as a set of sentences (called theoretical sentences) that contain only logical symbols, mathematical symbols, and the symbols of the theory (theoretical terms). The theoretical terms are connected to terms that refer to observable properties (observation terms) through sets of sentences that contain both theoretical and observation terms (correspondence rules). Both kinds of sentences are assumed to be in predicate logic of any order: Carnap and Hempel used higher order logic for the formalization of scientific theories (the former more often than the latter), and a fortiori neither demanded a restriction to first order logic (Lutz 2012, \$2). 
It is thus a historical puzzle that the Received View and (which is even more puzzling) syntactic approaches in general have been charged with demanding formalizations in first order logic. ${ }^{2}$ For instance, Suppes $(2002,3)$ criticizes the "standard sketch", by which he means the Received View, for the "strong tendency on the part of many philosophers to speak of the [theoretical sentences] as a logical calculus purely in syntactical terms" because theories like quantum mechanics "need to use $[\ldots]$ many results concerning the real numbers. Formalization of such theories in first-order logic is utterly impractical" (4). This argument rests essentially on the alleged restriction to first order logic because of "the success of second order logic to capture directly most all mathematical practice" (Leivant 1994, \$5.2) and might indeed be more natural a formalization of mathematical practice than set theory (Andrews 2002, xi-xiv).

Van Fraassen $(2014,281)$ criticizes the Received View's reliance on predicate logic in general, starting from Toulmin's exposition of the logical empiricists' project (Toulmin 1974, 602):

At its most grandiose, the ambition was to establish epistemological foundations $[\ldots]$ for positive scientific knowledge in its entirety. What $[\ldots]$ Peano and Russell had done by founding pure mathematics in formal logic $[\ldots]$ the advocates of unified science hoped to do for the whole of natural science. By adding further primitive terms, postulates, and correspondence rules, they hoped to incorporate all genuine branches of science into a single axiomatic edifice[.]

And van Fraassen $(2014,281)$ continues himself: “This revival of Leibniz' mathesis universalis is what Fred Suppe took on in his dissertation[.] It was equally what I saw as being replaceable[.]" It is clear from the above, however, that the Received View (as opposed to the logical empiricists' aim for the whole of science) does not require one language for all theories, but only one language for a theory and its correspondence rules. It was just the logical empiricists' ambition (and only at its most grandiose) to establish a unified language. Furthermore, a theory and its correspondence rules could be described in different languages-as long as there is at least one language of predicate logic, the assumptions of the Received View are fulfilled. ${ }^{3}$

Besides relying on predicate logic, the Received View assumes that the observation terms are given a direct semantic interpretation, which, through the correspondence rules and theoretical sentences, restricts the possible semantic interpretations of the theoretical terms (Carnap 1939, $\$ 24 ;$ Feigl 1970, 5-6). The resulting semantics can either be given informally by stating, for example, that the function " $\lg (x, t)$ ' designates the length in centimeters of the body $x$ at the time $t$ " (Carnap 1939, 58) or formally using, for example, Tarski semantics. The

2. In a recent example, Thompson (2007, 488-489) incorrectly claims a restriction to first order logic also for the foundations of the Received View (Whitehead and Russell, 1927/1929) and one of its applications (Woodger 1939).

3. In thank Richard Dawid for this point. 
latter would be natural because Carnap was an early defender of Tarski semantics (Carnap 1936). Such formal semantics for the Received View were independently developed by Przełęcki (1969), Suppe (1971), and Andreas (2010). That they all turned out to be equivalent or almost equivalent (Lutz 2014c) shows how strongly implied they are by Carnap's elucidations of the Received View.

It is clear that a set of sentences whose words are given no interpretation whatsoever cannot be about the world in any interesting sense. Formally, this point was made by Przełęcki $(1969,27-28)$ who, using an argument akin to the ones by Newman (1928) and Putnam $(1989,353)$, points out that a set of sentences whose terms have not been given an interpretation can at best determine the cardinality of its domain (cf. Lutz $2014 \mathrm{~d}, \mathbb{S}$ ). Thus the Received View and indeed any syntactic approach must, on pain of triviality, contain a semantic interpretation of some sort. This is why the Received View included semantic interpretations, as Suppe (1974a, II.E) also notes.

\section{Criticisms of Syntactic Approaches}

The criticisms discussed in the preceding section do not even apply to the Received View, let alone syntactic approaches in general. The question is now which criticisms do apply to syntactic approaches and thus what makes an approach syntactic. It is clear that the Received View has to be one specific syntactic approach. If it were not, the main question of the debate would become whom critics of syntactic approaches have been criticizing for 40 years.

A good starting point for the discussion of the criticisms of syntactic approaches is an overview of the fall of the Received View and syntactic approaches in general by Suppe $(2000,5103)$. He considers the following problems of the Received View severe:

1. Correspondence rules were a heterogeneous confusion of meaning relationships, experimental design, measurement, and causal relationships some of which are not properly parts of theories.

2. Symbolic logic is an inappropriate formalism.

3. Theories are not linguistic entities and thus theories are individuated incorrectly.

The first problem is clearly directed at the Received View in particular, not at syntactic approaches in general, since it only applies to syntactic approaches that rely on correspondence rules. Their use has many detractors (e. g., Suppes 2002, 7) and few defenders (Lutz 2014a, $\$ 4$ ). But it is clear that syntactic approaches in general may describe the relation between theories and observations without the use of correspondence rules. Indeed, Suppe (1974a, 108, n. 225) himself summarizes Suppes's competing semantic approach to the theory-observation relation 
"in terms of theories of which [Suppes's] models are realizations", that is, syntactically, and Turney (1990) and Lutz $(2014 \mathrm{a}, \mathbb{3}$ ) prove equivalences between their respective syntactic concepts and van Fraassen's semantic concept of empirical adequacy, which is also meant to describe the theory-observation relation. The Received View's related distinction between theoretical and observational terms has also been a focus of criticism (e.g., Putnam 1962; van Fraassen 1980, \$3.6), although it has some defenders (Suppe 1971, 1972; Chang 2005). As in the case of correspondence rules, however, syntactic approaches clearly do not have to rely on this distinction; the accounts by Turney (1990) and Lutz (2014a, $\$ 3$ ), for example, do not.

The second problem seems to apply to syntactic approaches in general. But Suppes argument for the severity of the second problem relies on the incorrect assumption that the Received View is restricted to first order logic: He refers to the Löwenheim-Skolem theorem (which holds only in first order logic) for arguing that syntactic approaches in general must lead to non-standard models, which makes symbolic logic an inappropriate formalism (Suppe 2000, s104). Clearly, the argument is therefore also not applicable to syntactic approaches in general (cf. Lutz 2014d, 1478-79). Van Fraassen's criticism of the use of predicate logic mentioned above relies on the logical empiricists' search for a mathesis universalis. As noted, the Received View does not require a single language for all scientific theories, and it is also clear that not all syntactic approaches require a single language for all scientific theories.

The third problem applies to all syntactic approaches, and will be the focus of my discussion. Suppe argues for this point as follows: Understanding 'propositions' "in the medieval sense of the term", that is, as "interpreted sentences of some particular language", Suppe (1974a, 204-5) claims that theories cannot be the propositions in which they are formulated because

quantum theory can be formulated equivalently as wave mechanics or as matrix mechanics; whichever way it is formulated, it is the same theory, though its formulations as wave mechanics will constitute a collection of propositions which is different from the collection of propositions resulting from its formulations as matrix mechanics.

Therefore van Fraassen $(1989,222)$ can claim that "in discussions of the structure of theories [language] can largely be ignored". But syntactic approaches always carry with them a language, which is why they must fail. As van Fraassen (1989, 221) writes: "In any tragedy, we suspect that some crucial mistake was made at the very beginning. The mistake, I think, was to confuse a theory with the formulation of a theory in a particular language". Halvorson $(2012,201-2)$ cites more claims by van Fraassen and others according to which the reliance on a particular language is a crucial mistake.

The question is how independent syntactic approaches can be from language. In the Received View (and in general), two theories are identified when they are described by logically equivalent sets of sentences. One could also go further 
and identify all analytically equivalent sets of sentence, that is, all sets whose equivalence follows logically from a set of meaning postulates (Lutz 2014d, $\mathbb{S}_{3}$ ). Halvorson $(2012,191)$ suggests the following equivalence relation between sets of sentences: Two sets $T$ and $T^{\prime}$ of sentences are definitionally equivalent if and only if they can both be extended by sets $\Delta$ and, respectively, $\Delta^{\prime}$ of explicit definitions such that $T \cup \Delta$ and $T^{\prime} \cup \Delta^{\prime}$ are logically equivalent. Mutual definability is another, more inclusive equivalence relation (cf. Andréka, Madarász, and Németi 2005). Two sets of sentences are mutually definable if and only if each set can be extended by explicit definitions such that it entails the other set. Both definitional equivalence and mutual definability lead to equivalence classes of sentences and thus to syntactic approaches. There is no obvious reason not to take other equivalence relations, like mutual interpretability (Hodges 1993, ch. 5), into account. And it is not obvious that wave and matrix mechanics are so different in their formulation that they are not definitionally equivalent, mutually definable, or mutually interpretable.

Summing up, the Received View did not rely on first order logic, nor did it distinguish between theories with logically equivalent axiomatizations. Syntactic approaches in general can rely on more general equivalence relations between a theory's axiomatizations, and they can do away with correspondence rules and the distinction between observational and theoretical vocabulary. This leaves two aspects of the Received View that make an approach syntactic: the use of predicate logic (possibly of higher order) and semantic interpretations (possibly formal). The restriction to predicate logic is necessary to not trivialize the notion of a syntactic approach. ${ }^{4}$ As van Fraassen $(1989,222)$ notes, "to present a theory, we must present it in and by language. That is a trivial point". The use of semantic interpretations is necessary to not trivialize all syntactic approaches, as the consideration by Przełęcki (1969, 27-28) shows. Thus an approach is syntactic if every theory is described by an interpreted set of sentences in higher order logic, where the interpretation does not have to be formal. Taking into account that different sets of sentences can be equivalent in one way or another, the following seems like the most plausible circumscription of syntactic approaches for the sequel:

Definition 1. An approach is syntactic if and only if in the approach every theory is described by an equivalence class of sets of sentences of higher order logic, where at least some of the sentences are interpreted.

This definition leaves open which equivalence relation generates the equivalence class, as different syntactic approaches can rely on different equivalence relations.

4. Carnap $(1956,64)$ in fact allows modal logic as well, but this will not be necessary for the following discussion. 


\section{Semantic Approaches}

The semantic approaches, even the formal ones, were never as unified as the logical empiricists' positions. In this section, I will survey those approaches that are important for the exchange between Halvorson, Glymour, and others, and suggest a general conception of semantic approaches that arguably underlies the debate.

\subsection{State Spaces}

In an outline of his version of the Semantic View (endorsed, for example, by Suppe 1974a, 222-23), van Fraassen (1970, 328-29) focuses on

the formal structure of nonrelativistic theories in physics [...]. A physical system is conceived of as capable of a certain set of states, and these states are represented by elements of a certain mathematical space, the state-space. [...] To give the simplest example, a classical particle['s] state-space can be taken to be Euclidean 6-space, whose points are the 6-tuples of real numbers $\left(q_{x}, q_{y}, q_{z}, p_{x}, p_{y}, p_{z}\right)$.

Van Fraassen $(1970,330)$ then distinguishes between laws of coexistence, laws of succession, and laws of interaction. In the non-statistical case ( $\$ 5.1)$, laws of coexistence select the physically possible subset of the state-space (330), laws of succession select, in the instantaneous state picture, the physically possible trajectories in the state-space (331), and laws of interaction at least in principle reduce to the above (332). The interpretation of physical theories relies on "elementary statements" $\ulcorner U(m, r, t)\urcorner$ formulating propositions "to the effect that a certain such physical magnitude $m$ has a certain value $r$ at a certain time $t$ " (328). Such a proposition is true if and only if the system is in state-space region $h(U)$, where $b$ is some satisfaction function. Finally, the "exact relation between $U(m, r, t)$ and the outcome of an actual experiment is the subject of an auxiliary theory of measurement, of which the notion of 'correspondence rule' gives only the shallowest characterization" (328). In van Fraassen's account, the state-space description is given in the metalanguage and semantically related to the elementary statements in the object language, which are then related to measurement outcomes via an auxiliary theory of measurement. Van Fraassen does not state whether the auxiliary theory is given in the object language and, relatedly, he is silent about the semantic interpretation of the elementary sentences. Van Fraassen's account of scientific theories therefore leaves out what the logical empiricists attempted to analyze: The connection of the formalism of the theory with the world. ${ }^{5}$

5. This is not an oversight: Van Fraassen $(1987,108)$ states that "the correct view about the meaning and understanding of newly introduced terms $[\ldots]$ makes no practical difference to philosophy of science, as far as one can tell. It is a good problem to pose to philosophers of language, and to leave them to it." 
This connection could thus be given (formally or informally) as in syntactic approaches.

Van Fraassen $(1970,326)$ notes that there "are natural interrelations between the two approaches" (his and the syntactic one) given by the possibility of describing a set of sentences by its models and a structure by the sentences of which it is a model. "These interrelations [...] would make implausible any claim of philosophical superiority for either approach." This claim of peaceful coexistence is gone in van Fraassen's exposition of constructive empiricism, his later approach to scientific theories and their relations to observations. Here van Fraassen $(1980,56)$ claims that the "syntactically defined relationships are simply the wrong ones" for the description of the relation between theory and observation. Worrall $(1984,71)$ points out the tension of this passage with van Fraassen's earlier remarks, and van Fraassen $(1989,212-213)$ replies:

$[\mathrm{W}] \mathrm{e}$ must advance the conception of natural language as not being constituted by any one realization of any such logical skeleton [of predicate logic]. [...] In my opinion, it would be a poor choice to try and describe a whole language in which a given theory can be formulated. The reason is that descriptions of structure in terms of satisfaction of sentences is much less informative than direct mathematical description. [Instead] we may choose a very small fragment, such as what I have called the fragment of elementary statements. [Their syntactic form is trivial] and therefore the semantic study alone has some significance.

And in an endnote, $(365$, n. 34 ; cf. van Fraassen 1987, 121) adds: "It should be added however that I soon found it much more advantageous to concentrate on the propositions expressible by elementary statements, rather than on the statements themselves. [...] At later points there is not even a bow in the direction of syntactic description." Having started out with an account of scientific theories that includes an object language (for elementary sentences) and a metalanguage (for the description of the state-space), van Fraassen has thus arrived at an account that contains only what used to be the metalanguage. Since 'object language' and 'metalanguage' have only a relative sense (cf. Tarski 1944, \$9), van Fraassen's account at this point is simply phrased in a single language. Since natural language is interpreted, the only reason why van Fraassen's account of scientific theories is not syntactic is thus that he does not assume that theories are formalized in predicate logic, but rather informally in natural language and the semi-regimented natural language of mathematics.

As noted, higher order logic can capture mathematical practice quite directly (Leivant 1994, 260). Thus as far as the mathematical component of scientific theories is concerned (that is, the component described by van Fraassen's account), it is not clear why there should be a principled problem with the use of higher order logic as opposed to informal mathematics. It may seem that syntactic approaches still have the disadvantage of dealing with equivalence classes of sets of 
sentences, but the state-space approach is in the same position: The same scientific theory can be described in different state-spaces, or in the same state-space but with differently formulated restrictions. Therefore the state-space approach also has to deal with equivalence classes of sentences, albeit not necessarily in predicate logic.

\subsection{Structures}

As van Fraassen notes, his later approach to scientific theories and their relations to observations, constructive empiricism, does not rely on elementary statements. The formal aspect of constructive empiricism is phrased completely in terms of model theory: A theory is given by a family of structures (called 'models of the theory'), each appearance is given by a single structure, and a theory is empirically adequate if and only if all appearances are isomorphic to specially designated substructures ("empirical substructures") of a member of the family of structures (van Fraassen 1980, \$3.9; cf. Lutz 2014d, \$3.1). It is not obvious how this approach relates to van Fraassen's state-space approach: Elementary sentences cannot be embedded in state-spaces in the normal sense of the word, and the embeddability of the propositions expressed by elementary sentences in state-spaces is even more questionable as long as 'proposition' has not received a mathematical definition. But with his new formalism, van Fraassen $(1989,366)$ can claim independence from the syntax of predicate logic, because he considers structures to be so independent:

The impact of Suppes's innovation [i. e., switching to models] is lost if models are defined, as in many standard logic texts, to be partially linguistic entities, each yoked to a particular syntax. In my terminology here the models are mathematical structures, called models of a given theory only by virtue of belonging to the class defined to be the models of that theory.

A standard text in which models are "yoked to a particular syntax" is the textbook by Chang and Keisler (1990, $\$ 1.3$ ), where structures are defined as pairs $\langle A, \mathscr{I}\rangle$ of a non-empty set $A$ of objects (a domain) and an interpretation $\mathscr{I}$, which is a mapping from each $n$-ary relation symbol to an $n$-ary relation $R \subseteq A^{n}$ on $A$, each $m$-place function symbol $F$ to an $m$-place function $G: A^{m} \longrightarrow A$ on $A$, and each constant symbol $c$ to a constant $x \in A$. From the notation of the relation and constants on $A$ it is obvious that these are extensional concepts such that, for instance, $R=R^{\prime}$ when for all $x, x \in R$ if and only if $x \in R^{\prime}$. Relations with the same extension are identical, while there can be two different relation symbols with the same extensions of their interpretations. Hodges $(1993,1-4)$ explicitly stresses this reliance on a vocabulary.

It is thus somewhat unfortunate that in his discussion of the role of language, van Fraassen $(2014,277)$ refers to Chang and Keisler (1990) for the detailed definition of 'structure'. For their reliance on a vocabulary is essential and cannot 
be avoided by identifying "the structure $[\ldots]$ as the range of the interpretation" (van Fraassen 2014, n. 1): If, for instance, a structure $\langle A, \mathscr{I}\rangle$ contains an interpretation solely for multiple relation symbols $P_{i}, i \in I$, and maps all of them to the same $R$, taking its image would lead to $\langle A,\{R\}\rangle$, so that only one relation would be left. However, van Fraassen also refers to Robinson (1963, 9-10), who uses an informal version of a conception of 'structure' that is used by Bell and Slomson $(1974, \mathbb{\$} 3.2)$. They define a relational structure $\mathfrak{A}$ as a pair $\left\langle A,\left\{R_{i}\right\}_{i \in I}\right\rangle$ of a domain $A$ and a family (an indexed set) $\left\{R_{i}\right\}_{i \in I}$ of extensional relations. ${ }^{6}$ Similarly, Robinson $(1963,9)$ does "not identify the relations as such with sets of ordered $n$ ples of individual objects of [structure] $M$ so that two relations may hold at the same $n$-ples." That is, Robinson uses non-extensional relations that correspond to pairs of (extensional) relations and their index in the formalism of Bell and Slomson. Robinson then interprets the vocabulary by a bijection between the vocabulary and the set of non-extensional relations or, in the formalism of Bell and Slomson $(1974, \mathbb{3} .2)$, by a bijection between the vocabulary and the index set $I$ of the family of relations. ${ }^{7}$

In the following, I will call structures formalized via a vocabulary 'labelled structures' and structures formalized via an index set 'indexed structures'. A set containing the symbols of a structure and their associated types is a signature. Note that labelled structures uniquely determine a signature, while indexed structures only determine the types of the signature, not the associated symbols. The difference between indexed and labelled structures is of central importance for van Fraassen (as detailed above) and others (e. g., French and Ladyman 1999, 11415). It marks the difference between a notion of structure that is independent from any vocabulary of predicate logic and a notion of structure that uniquely determines such a vocabulary.

Van Fraassen $(2014,280)$ also cites a text by Suppes $(1957, \$ 12.2)$ to show that structures can be defined independently of a vocabulary. Here, structures are written as tuples containing a domain and relations, functions, and constants. On the face of it, such a tuple has neither a vocabulary nor an index set, but when interpreted as a labelled structure, the vocabulary is implicitly understood (Hodges 1993, 4), and interpreted as an indexed structure, the position in the tuple takes the role of the index set, so that $\left\langle A, R_{1}, \ldots, R_{n}\right\rangle$ can be read as $\left\langle A,\left\{R_{i}\right\}_{i \in\{1, \ldots, n\}}\right\rangle$ (cf. Lutz 2014d, 1480-84). Thus one cannot say that "reducing a [labelled] model to a bare set-theoretical $n$-tuple would be akin to reducing a function $f: A \longrightarrow B$ to its image $f(A)$. Obviously, the function $f$ contains more data than its image $f(A)$, and in exactly the same way, a model contains more data than just the resulting set-theoretical structure" (Halvorson 2013, 477). For,

6. It is clear from their use of the definition that Bell and Slomson (1974) intend ' $\left\{R_{n} \mid n \in \omega\right\}$ ' to be a family (rather than the set of the family's elements).

7. To be precise, Robinson assumes an injective mapping from the relations to the vocabulary, which has the advantage that a structure can interpret vocabularies with symbols that do not occur in the structure. Bell and Slomson $(1974, \$ 3.2)$ use a family of symbols and a family of relations with the same index set, which leads to a natural bijection. 
as discussed in connection with van Fraassen's remark above, an indexed structure contains more information than the image of an interpretation in the case of a labelled structure. In general, if $\mathscr{I}: P_{i} \mapsto R_{i}$, then taking the image of $\mathscr{I}$ leads to $\left\langle A,\left\{R_{i} \mid i \in I\right\}\right\rangle \neq\left\langle A,\left\{R_{i}\right\}_{i \in I}\right\rangle$. Specifically, it can be the case that $\left\langle A,\left\{R_{i}\right\}_{i \in I}\right\rangle \neq\left\langle B,\left\{S_{i}\right\}_{i \in I}\right\rangle$ while $\left\langle A,\left\{R_{i} \mid i \in I\right\}\right\rangle=\left\langle B,\left\{S_{i} \mid i \in I\right\}\right\rangle$ (the opposite case is impossible).

(Van Fraassen $(2014,277)$ calls the combination of an indexed structure with an appropriate interpretation a 'model', and can thus state that "a model is [...] a structure with an interpretation". But it is unclear why van Fraassen would want to use this definition of 'model' for his semantic approach, since, save for some renaming of indexes, the definition makes a model into nothing else but a labelled structure. Furthermore, his claim that according to this definition "a model is [...] an interpreted structure" is still highly misleading, for it is not the structure that is interpreted, but the vocabulary; a model in this sense contains an interpreting structure.)

\subsection{A Fairly General Account of Semantic Approaches}

To avoid the yoke of a vocabulary, semantic approaches should rely on indexed rather than labelled structures. This is the first aspect of the fairly general account of semantic approaches that I want to develop now. Its second aspect springs from van Fraassen's observation that the structures describing a theory are themselves "describable only up to structural isomorphism" (van Fraassen 2008, 238; cf. van Fraassen 2002, 22). Furthermore, often not all of a theory's structures are isomorphic to each other, because many theories are not categorical. Thus the second aspect of semantic approaches is that a theory is described by a class of indexed structures, not a single structure. A theory can also typically be described by different classes of structures. In the case of van Fraassen's state-spaces, different state-spaces for the same theory are not isomorphic, and when Hendry and Psillos (2007, 137, my emphasis) state in connection with the equivalence of matrix and wave mechanics that "a (semantic) model of one could be turned into a model of the other", they effectively point out that the structures are not the same (cf. Halvorson 2012, 188). Finally, it is clear that a theory cannot consist solely of purely formal structures, since they have to connect to empirical results in some way (see also Lutz 2014d, \$4). In publications relying on semantic approaches, this connection is typically not called 'interpretation' as in syntactic approaches, but 'representation' (e.g., Suppes 2002). Thus for the sequel, it is appropriate to describe semantic approaches as follows:

Definition 2. An approach is semantic if and only if in the approach every theory is described by an equivalence class of classes of indexed structures, where at least some of the structures represent.

Often, a class of indexed structures is described using a set theoretical or Suppes predicate (see Suppes 2002, \$2.3), which is a set theoretic description of 
the domains, relations, functions, and constants of the structures as well as the formulas the relations, functions, and constants have to fulfill. It is clear that, as in the case of state-spaces, the same class of structures can be described in different ways, so that semantic approaches fulfilling definition 2 also have to deal with equivalence classes of sentences.

At this point, it might seem as if semantic approaches are nothing but specific syntactic approaches that, unlike syntactic approaches in general, are wedded to the use of the language of set theory. The reasoning goes as follows: The set theoretical sentences describing the Suppes predicate of a theory can be given either in a set theory axiomatized in higher order logic, in a set theory axiomatized in first order logic, or in informal, naive set theory. First order set theory is essentially higher order logic (see, e.g., Väänänen 2001, \$2) and thus if the set theoretical sentences are axiomatized at all, the theory described by the Suppes predicate is described in higher order logic and thus according to a syntactic approach. For semantic approaches to be something other than specific syntactic approaches, the set theoretical sentences describing the Suppes predicate must therefore be given in naive set theory. As already noted in connection with van Fraassen's disavowal of any syntactic leanings $(\mathbb{4} 4.1)$, the difference is that between predicate logic and the semi-regulated language of mathematics. But as Suppes (2002, 30) writes:

The only important distinction between axiomatic and naive set theory is that in axiomatic set theory one continually must consider questions about the existence of sets[.] Although questions about the existence of sets shall not be considered explicitly here, it is a simple and routine matter to embed everything that is said $[\ldots]$ within some well-defined axiomatic set theory[.]

It seems, then, that only a simple and routine matter keeps semantic approaches from being specific syntactic approaches.

If the preceding argument were sound, my thesis that there is no essential difference between syntactic and semantic approaches would have been established, and I could end here. But the argument leaves out the interpretation of syntactically described theories and the representation relation of semantically described theories. In analogy to the interpretation of syntactic approaches, one can leave the representation relation informal; in that case, there is indeed no principled difference between syntactic and semantic approaches. But the predominant formal approaches assume that a structure represents via an isomorphism or some generalization thereof, for example an embedding (cf. Halvorson 2012, 185). Like the use of Tarski semantics, this demand requires that objects in the world can be members of sets, and that it is possible to map from abstract objects like numbers to objects in the world and sets thereof (Lutz 2014d, $\$ 4$ ). But the isomorphism between two structures is different from the interpretation of a set of sentences by a structure, simply on formal grounds. An isomorphism relates objects in one domain to objects in another, and, for instance, elements of one relation to 
elements of another. An interpretation, on the other hand, relates primitive relation symbols to (Cartesian products of) classes of objects of some domain. Thus the difference between interpretations and representations distinguishes between syntactic and semantic approaches. Specifically, a theory described by a set of sentences as in a syntactic approach can be formally interpreted by a structure as given in semantic approaches, but a structure is not isomorphic to a set of sentences. Note, however, that this does not mean that either approach is easier to connect to objects in the world. In fact, an isomorphism relation between the world and an indexed structure can be used to construct an interpretation of a set of sentences, and an interpretation of a set of sentences by the world can be used to construct an isomorphism relation to a structure (Lutz 2014d, \$4).

There are many semantic approaches in the wider sense that are completely unrelated to the above discussion, namely those that interpret 'model' not in the sense of model theory but as 'scientific model'. As Frigg and Hartmann (2008, \$4.1) put it: "Although different [semantic approaches] assume a different notion of model $[\ldots$,$] they all agree that models are the central unit of scientific theoriz-$ ing." In other words, semantic approaches in the wider sense share with semantic approaches in the narrow sense only the commitment to calling the central units of scientific theorizing 'models'. Suppes (1960) provided an influential argument that the meaning and use of 'model' in the sciences is the same as in model theory (cf. Suppes 2002, \$2.1). But his argument at best establishes that the formal aspect of scientific models can be reformulated in set theory, and it relies on the tacit (and problematic) identification of set theoretic descriptions with descriptions in model theory (Lutz 2012, 97-99). Hence semantic approaches in the wider sense may very well be as close to syntactic approaches as to semantic approaches, and the relevance of scientific models for the debate between the two approaches may very well be nil. Thus the claim by van Fraassen $(2014,278)$ that "models in the sciences are not exactly what are called models in meta-mathematics" is something of an understatement.

\section{Criticism of Semantic Approaches}

Criticisms of semantic approaches usually have been limited to very specific approaches (e.g., Suárez and Cartwright 2008, \$5) or have been aimed at all attempts at formalization (see Halvorson 2012, \$7). The critique by Halvorson (2012) therefore stands out in that it criticizes specifically semantic approaches. At the core of Halvorson's critique lies the question of theory individuation. In a reversal of Suppe's criticism, Halvorson argues that semantic approaches incorrectly distinguish between different formulations of the same theory, that is, make distinctions without a difference. But Halvorson goes further and argues that semantic approaches also fail to distinguish theories that are different. 


\subsection{An Inability to Distinguish Between Different Theories}

At the core of Halvorson's argument that semantic approaches identify theories that are different lies his notion of isomorphism for semantic approaches. It is explicated by Glymour $(2013,291)$ and accepted by Halvorson $(2013,476)$ in roughly the following form:

Definition 3. Two structures $\left\langle A, R_{1}, \ldots, R_{n}\right\rangle$ and $\left\langle B, S_{1}, \ldots, S_{n}\right\rangle$ are $H$-isomorphic just in case there is a bijection $j: A \longrightarrow B$ and a bijection $k:\left\{R_{i}\right\}_{i \in\{1, \ldots, n\}} \longrightarrow$ $\left\{S_{i}\right\}_{i \in\{1, \ldots, n\}}$ such that $\left\langle a_{1}, \ldots, a_{n}\right\rangle \in R_{i}$ if and only if $\left\langle j\left(a_{1}\right), \ldots, j\left(a_{n}\right)\right\rangle \in k\left(R_{i}\right){ }^{8}$

This definition is not obviously uncharitable; in fact, it is the definition developed and endorsed by Turney (1990, def. 2) in his explication of van Fraassen's notion.

Halvorson now uses the notion of $\mathrm{H}$-isomorphism to argue that semantic approaches fail to distinguish different theories. To do this, he assumes that in semantic approaches a theory is described by an equivalence class of classes of structures, as is also assumed in definition 2. And he assumes that a class of structures is equivalent to another if and only if the two are pointwise H-isomorphic, that is, there is a bijection $F$ from one to the other such that $F(\mathfrak{A})$ is $\mathrm{H}$-isomorphic to $\mathfrak{A}$ (Halvorson 2012, 190). The argument Halvorson $(2012,192)$ uses for showing that pointwise $\mathrm{H}$-isomorphisms identify different theories can be slightly shortened as follows:

Claim 1. Let $T$ and $T^{\prime}$ have the same signature containing one-place first-order predicates $P_{1}$ and $P_{2}$. Let $T=\{\exists x \forall y(x=y)\}$ and $T^{\prime}=T \cup\left\{\forall x\left(P_{1} x \rightarrow P_{2} x\right)\right\}$. Then $T$ and $T^{\prime}$ are not logically equivalent, but they have H-isomorphic models.

Proof. Every model of $T^{\prime}$ is (and thus is H-isomorphic to) a model of $T$. Without loss of generality, assume that for $T^{\prime}$, the syntactic predicate $P_{1}$ is interpreted by semantic relation $S_{1}$ and $P_{2}$ by $S_{2}$. Then the models of $T^{\prime}$ are given by the class $\left\{\left\langle\{a\}, S_{1}, S_{2}\right\rangle \mid S_{1} \subseteq S_{2}, a\right.$ arbitrary $\}$. Now let $\mathfrak{A}=\left\langle\{a\}, R_{1}, R_{2}\right\rangle$ be any model of $T$. If $a \in R_{1}, R_{2}$ or $a \notin R_{1}, R_{2}$, then $\mathfrak{A}$ is a model (and thus $\mathrm{H}$-isomorphic to a model) of $T^{\prime}$. If $R_{i}=\{a\}$, and $R_{j}=\varnothing,\{i, j\}=\{1,2\}$, choose $k$ of definition 3 so that $k\left(R_{j}\right)=S_{1}$ and $k\left(R_{i}\right)=S_{2}$.

Thus two theories that can be distinguished in syntactic approaches cannot be distinguished in semantic approaches, making semantic approaches less expressive. Since furthermore $T$ and $T^{\prime}$ are also intuitively different, semantic approaches are intuitively inadequate.

Glymour's response to Halvorson's example is that pointwise H-isomorphism is the wrong equivalence relation between classes of structures, and specifically that $\mathrm{H}$-isomorphism is the wrong relation between structures. Instead, Glymour $(2013,291)$ argues and Halvorson $(2013,476)$ agrees, semantic

8. Halvorson omits $k$ and instead writes ' $\left\langle j\left(a_{1}\right), \ldots, j\left(a_{n}\right)\right\rangle \in S_{j}$ ', where $j$ is meant to stand for $\pi(i)$, with $\pi$ a permutation on $\{1, \ldots, n\}$ (email from 27 May 2014). 
approaches must rely on labelled structures and the standard model theoretic notion of isomorphism for labelled structures. Specifically, the existence of an isomorphism between two structures must entail their elementary equivalence, as it does in standard model theory. The use of labelled structures, of course, forces the semantic view under the yoke of language, since it determines a specific signature of predicate logic (cf. Glymour 2013, 288-289; Halvorson 2013, 476).

But labelled structures and the associated notion of isomorphism are not necessary to block Halvorson's example. The example is also blocked by indexed structures and their associated notion of isomorphism. For relational first order structures, it is given by Bell and Slomson $(1974,73)$ and can be written as follows:

Definition 4. An isomorphism from an indexed structure $\mathfrak{A}=\left\langle A,\left\{R_{i}\right\}_{i \in I}\right\rangle$ to $\mathfrak{B}=\left\langle B,\left\{S_{i}\right\}_{i \in I}\right\rangle$ is a bijection $b$ from $A$ to $B$ such that for any $n$-ary relation $R_{i}$, $i \in I$ and all $a_{1}, \ldots, a_{n} \in A,\left\langle a_{1}, \ldots, a_{n}\right\rangle \in R_{i}$ if and only if $\left\langle h\left(a_{1}\right), \ldots, b\left(a_{n}\right)\right\rangle \in S_{i}$.

The difference to $\mathrm{H}$-isomorphisms (definition 3 ) is clear: An $\mathrm{H}$-isomorphism is an isomorphism if and only if $k\left(R_{i}\right)=S_{i}$ for all $1 \leq i \leq n$. And this difference suffices for blocking Halvorson's argument against pointwise $\mathrm{H}$-isomorphisms:

Claim 2. Let $T$ and $T^{\prime}$ have the same signature containing one-place first-order predicates $P_{1}$ and $P_{2}$. Let $T=\{\exists x \forall y(x=y)\}$ and $T^{\prime}=T \cup\left\{\forall x\left(P_{1} x \rightarrow P_{2} x\right)\right\}$. Then the classes of models of $T$ and $T^{\prime}$ are not pointwise isomorphic.

Proof. In $T$, let $P_{1}$ be assigned to $R_{i}$ and $P_{2}$ assigned to $R_{j}$, with $\{i, j\}=\{1,2\}$. Then the class of models of $T$ contains every structure $\left\langle\{a\}, R_{1}, R_{2}\right\rangle$ with a singleton domain. In $T^{\prime}$, let $P_{1}$ be assigned to $S_{k}$ and $P_{2}$ assigned to $S_{l}$. Then the class of models of $T^{\prime}$ contains all and only structures with a singleton domain and $S_{k} \subseteq S_{l}$, and thus for domain $\{b\}$ no structures with $S_{k}=\{b\}$ and $S_{l}=\varnothing$. There are then two possibilities. If $i=k$ and $j=l$, there is a $T$-model with $R_{i}=\{a\}$ and $R_{j}=\varnothing$ but no isomorphic $T^{\prime}$ model. If $j=k$ and $i=l$, there is a $T$-model with $R_{j}=\{a\}$ and $R_{i}=\varnothing$ but no isomorphic $T^{\prime}$ model.

Note also that two isomorphic indexed structures are only elementary equivalent if they have the same vocabulary, with each symbol mapped to the same index of the index set of the structure's family.

With the definition of isomorphism for indexed structures, it is easy to define the notion of embedding that is so central to constructive empiricism: ${ }^{9}$

Definition 5. An embedding of an indexed structure $\mathfrak{A}=\left\langle A,\left\{R_{i}\right\}_{i \in I}\right\rangle$ in $\mathfrak{B}=$ $\left\langle B,\left\{S_{i}\right\}_{i \in I}\right\rangle$ is an isomorphism from $\mathfrak{A}$ to a substructure $\left\langle C,\left\{S_{i} \cap C^{n_{i}}\right\}_{i \in I}\right\rangle$ of $\mathfrak{B}$, where $C \subseteq B$ and $n_{i}$ is the arity of $S_{i}$.

9. Halvorson's argument that semantic approaches cannot rely on the model theoretic notion of embedding is discussed in appendix A. 
It remains to be seen why Glymour and Halvorson both assume that there cannot be an appropriate notion of isomorphism for structures without a unique signature. An argument by Glymour $(2013,288)$ for the need for labelled structures provides a hint:

Suppose a theory has multiple models, one of which is $\left\langle D_{1} ; \varphi, \chi, \varphi\right\rangle$, and there is a data model that maps three measured quantities to the three relations. To predict what would happen were the conditions of measurement changed, another model, say $\left\langle D_{2} ; \tau, \delta, \omega\right\rangle$, must be chosen and the measured quantities mapped to relations of that model, but the radical semantic view has insufficient resources to specify which relations among $\tau, \delta, \omega$ in this second model correspond to $\varphi, \chi, \varphi$, respectively.

However, $\left\langle D_{1} ; \varphi, \chi, \varphi\right\rangle$ and $\left\langle D_{2} ; \tau, \delta, \omega\right\rangle$ are tuples, and hence there is a perfectly fine way to specify which relations among $\tau, \delta, \omega$ correspond to which relations among $\varphi, \chi, \varphi$ : The first relation in the one, $\varphi$, corresponds to the first relation in the other, $\tau$, and analogously for the second and third relations.

I surmise that while Glymour is explicit about his use of a non-standard notion of isomorphism in his discussion, he implicitly assumes a non-standard notion of structure as well. His notion does not contain an ordering of the relations, but only their number:

Definition 6. The $H$-structure corresponding to an indexed structure $\left\langle A,\left\{R_{i}\right\}_{i \in I}\right\rangle$ is $\left\langle A,\left[R_{i} \mid i \in I\right]\right\rangle$.

$\left[R_{i} \mid i \in I\right]$ is the multiset resulting from the family $\left\{R_{i}\right\}_{i \in I} \cdot{ }^{10} \mathrm{~A}$ multiset is like a set, except that elements can occur any finite number of times (Blizard 1989). The notion of an H-structure fits very well with Glymour's argument for the need for labelled structures: As long as structures are expressed by tuples (or families), each relation in the structure $\left\langle D_{1}, \varphi, \chi, \varphi\right\rangle$ is associated with exactly one relation of the structure $\left\langle D_{2}, \tau, \delta, \omega\right\rangle$. It is only when each structure is expressed by a domain and a multiset, $\left\langle D_{1},[\varphi, \chi, \varphi]\right\rangle$ and $\left\langle D_{2},[\tau, \delta, \omega]\right\rangle$, that the correspondence is lost. And this loss of correspondence is analogous to the loss of correspondence incurred through the change from isomorphisms to H-isomorphisms:

Claim 3. Structure $\mathfrak{A}$ is $H$-isomorphic to $\mathfrak{B}$ if and only if there are a structure $\mathfrak{A}^{\prime}$ with the same corresponding $H$-structure as $\mathfrak{A}$ and a structure $\mathfrak{B}^{\prime}$ with the same corresponding $H$-structure as $\mathfrak{B}$ such that $\mathfrak{A}^{\prime}$ is isomorphic to $\mathfrak{B}^{\prime}$.

Proof. Two structures $\left\langle A,\left\{R_{i}\right\}_{i \in I}\right\rangle$ and $\left\langle A^{\prime},\left\{R_{i}^{\prime}\right\}_{i \in I}\right\rangle$ have the same corresponding H-structure if and only if $A=A^{\prime}$ and there is a bijection $k:\left\{R_{i}\right\}_{i \in I} \longrightarrow$ $\left\{R_{i}^{\prime}\right\}_{i \in I}$ such that $k\left(R_{i}\right)=R_{j}^{\prime}$ for some $j \in I$. Thus it is straightforward to show

10. I follow Hein $(2003,29)$ in designating multisets by square brackets. 
that $f$ is an isomorphism from $\mathfrak{A}$ to $\mathfrak{B}$ if and only if $f$ is an H-isomorphism from some $\mathfrak{A}^{\prime}$ to some $\mathfrak{B}^{\prime}$ that have the same corresponding $\mathrm{H}$-structures as $\mathfrak{A}$ and $\mathfrak{B}$, respectively.

Thus for two $\mathrm{H}$-structures $\mathfrak{A}$ and $\mathfrak{B}$, the claim that some structures corresponding to $\mathfrak{A}$ and $\mathfrak{B}$ are isomorphic is equivalent to the claim that some structures corresponding to $\mathfrak{A}$ and $\mathfrak{B}$ are $\mathrm{H}$-isomorphic. Phrased more casually, the notion of isomorphism is indistinguishable from the notion of $\mathrm{H}$-isomorphism after the move from structures to H-structures. This seems to be the most plausible justification of Glymour's and Halvorson's claim that without a language, only $\mathrm{H}$-isomorphisms are possible: The claim follows from the assumption that without language, only $\mathrm{H}$-structures are possible.

\subsection{Distinctions Without a Difference}

Halvorson not only argues that semantic approaches identify different theories, but also that semantic approaches make distinctions without a difference. Underlying his argument is the assumption that the equivalence relation for syntactic approaches is definitional equivalence (Halvorson 2012, 191). As Halvorson (2013, 193-94) notes, the theory of groups can be given a syntactic description with a signature containing a first order two-place relation symbol 'o' and a first order constant symbol ' $e$ ', but also a definitionally equivalent syntactic description with a signature containing only ' 0 '. The classes of indexed models of these descriptions are not pointwise $\mathrm{H}$-isomorphic.

Glymour's response is straightforward: The equivalence classes of semantic approaches can also be taken to be determined by definitional equivalence, which in first order model theory can be phrased equivalently in terms of structures using coalescence (Glymour 2013, 292-93). Glymour again assumes labelled structures for his argument, but the concept of coalescence can also be defined using indexed structures. ${ }^{11}$ There is also another, very cheap way of defining definitional equivalence without reference to a vocabulary: An explicit definition of $n$-place relation symbol $P$ has the form $\left\ulcorner\forall x_{1} \ldots x_{n}\left[P x_{1} \ldots x_{n} \leftrightarrow \varphi\left(x_{1}, \ldots, x_{n}\right)\right]\right\urcorner$, where $x_{1}, \ldots, x_{n}$ are pairwise different variables and $\varphi$ is some formula with $n$ free variables. If the relation $R$ is assigned to $P$, then the model theoretic definition of truth entails a corresponding set theoretic condition $\forall a_{1}, \ldots, a_{n}\left[\left\langle a_{1}, \ldots, a_{n}\right\rangle \in\right.$ $\left.R \Leftrightarrow \varphi^{*}\left(a_{1}, \ldots, a_{n}\right)\right]$, where $\varphi^{*}$ is the set theoretic formula that corresponds to $\varphi$ (cf. Przełęcki 1969, 14-15; Schurz 2014, 95). ${ }^{12}$ This procedure is also possible in higher order logic. The sets $\Delta$ and $\Delta^{\prime}$ used to establish definitional equivalence ( $\left.T \cup \Delta \models T^{\prime} \cup \Delta^{\prime}\right)$ of two theories $T$ and $T^{\prime}$ can thus be translated into set

11. These definitions rely on the notions of expansion and reduct: An indexed structure $\mathfrak{A}=$ $\left\langle A,\left\{R_{i}\right\}_{i \in I}\right\rangle$ is a reduct of $\mathfrak{B}=\left\langle B,\left\{S_{j}\right\}_{j \in J}\right\rangle$ if and only if $A=B, I \subseteq J$, and $R_{i}=S_{i}$ for all $i \in I \cdot \mathfrak{A}$ is an expansion of $\mathfrak{B}$ if and only if $\mathfrak{B}$ is a reduct of $\mathfrak{A}$.

12. Definitions of function and constant symbols require additional considerations that are technically important but do not change the conclusions of this discussion. 
theoretic conditions on all expansions of the elements of two classes of indexed structures $\mathbf{T}$ and $\mathbf{T}^{\prime}$. If these expansions lead to the same class of structures, $\mathbf{T}$ and $\mathbf{T}^{\prime}$ can be treated as in the same equivalence class. The classes of models of two syntactic theories $T$ and $T^{\prime}$ are so equivalent if and only if the two syntactic theories are definitionally equivalent. Being somewhat more subtle, one could define set theoretic restrictions on the expansions of structures in $\mathbf{T}$ and $\mathbf{T}^{\prime}$ so that each structure can be expanded in exactly one way. If there is then a class of structures that fulfills these restrictions and has $\mathbf{T}$ and $\mathbf{T}^{\prime}$ as reducts, call that class the common unique expansion class of $\mathbf{T}$ and $\mathbf{T}^{\prime}$.

Claim 4. If $T$ and $T^{\prime}$ are definitionally equivalent, the classes of their models have a common unique expansion class. If two classes $\mathbf{T}$ and $\mathbf{T}^{\prime}$ of indexed structures are models of first order theories (finite higher order theories) $T$ and $T^{\prime}$, respectively, and have a common unique expansion class that is the class of models of some first order theory (finite higher order theory) $T^{*}$, then $T$ and $T^{\prime}$ are definitionally equivalent.

Proof. An explicit definition of a symbol uniquely determines the symbol's extension in any expansion (Hodges 1993, 61). Since the restrictions on the expansions can be expressed in set theory, the first part of the claim follows. For the second part, note that by assumption every model of $T$ and $T^{\prime}$, respectively, can be expanded to a model of $T^{*}$, and this expansion is unique. For first order logic, it follows from Beth's theorem that $T^{*}$ entails explicit definitions for all symbols not in $T$ or in $T^{\prime}$. For finite theories of higher order logic, this was shown by Tarski (1935, theorem 3; cf. Leivant 1994, \$5.1).

Halvorson $(2013,475)$ agrees with Glymour's claim that with labelled structures, the corresponding notion of isomorphism, and definitional equivalence, Halvorson's examples of incorrect identifications of theories are blocked. But with definitional equivalence as the identity criterion for theories, so Halvorson $(2013,478)$, one central application of semantic approaches, structural realism, is in trouble:

Recall that some structural realists want to subscribe to the view that:

(P1) To believe a theory $T$ means to believe that the world is isomorphic to a model of $T$.

But structural realists should also be committed to the claim that:

(P2) There is no good reason to believe $T$ over an equivalent theory $T^{\prime}$.

$[\ldots]$ But P1 and P2 [...] entail that believing $T$ means believing that the world is isomorphic to a model of $T$ but also that the world is isomorphic to a model of $T^{\prime}$. But nothing is isomorphic both to a model of $T$ and to a model of $T^{\prime}$. 
In this argument, Halvorson assumes that the models of $T$ are the structures in which $T$ is true, that is, he assumes that 'model of $T$ ' is understood as in model theory. With definition 2 and definitional equivalence as the equivalence relation, however, the models of $T$ are those in which $T$ or any $T^{\prime}$ definitionally equivalent to $T$ is true. (Hence in semantic approaches it would be advantageous to speak of the models that describe $T$ to distinguish this notion from the notion of 'model of $T$ ' in model theory.) Thus Halvorson's argument does not establish that structural realism is in trouble, but rather the following: If structural realists believe a theory $T$, the structures that they can ascribe to the world are not restricted to the models of $T$, but to the models that describe $T$, that is, models of theories $T^{\prime}$ that are definitionally equivalent to $T$.

\subsection{H-structures, Indexed Structures, and Labelled Structures}

Glymour and Halvorson agree that $\mathrm{H}$-isomorphisms (and thus $\mathrm{H}$-structures) are insufficient for an analysis of scientific theories. They also agree that therefore, semantic approaches must rely on labelled structures, which include a vocabulary and therefore a full language. But this poses a problem, as Halvorson $(2013,475)$ observes, for "if we add language to the semantic view, then we have a syntactic view" ${ }^{13}$ I have argued above that the use of labelled structures in a semantic approach indeed makes it into a syntactic approach, but proponents of semantic approaches can very well disagree with Glymour's and Halvorson's claim that because $\mathrm{H}$-structures do not suffice for the description of theories, semantic approaches have to rely on labelled structures. As argued above, the semantic view relies on indexed structures, and proponents of semantic views have put a lot of weight on the distinction between indexed and labelled structures in their arguments for the superiority of semantic approaches (see $\$ 4.2$ ).

How important, then, is the distinction? I claim that formally, there is no relevant distinction at all and thus that Glymour and Halvorson are correct in their conclusion (cf. Lutz 2014d, 1480). The case is especially obvious for relational structures: Here the labelled structure is given by a pair of a domain and an interpretation of all relation symbols:

$$
\langle A, \mathscr{J}\rangle \text { with } \mathscr{J}: \mathscr{V} \longrightarrow\left\{R_{i} \mid i \in I\right\}
$$

An indexed relational structure is given by a pair $\left\langle A,\left\{R_{i}\right\}_{i \in I}\right\rangle$, containing a domain and a family of relations. The standard definition of a family $\left\{R_{i}\right\}_{i \in I}$ is that of a mapping $f: I \longrightarrow\left\{R_{i} \mid i \in I\right\}$ from the family's index set to the set of relations (Bourbaki 1968, 81-82). Substituting the definiens for the definiendum, the indexed structure is therefore given by

$$
\langle A, f\rangle \text { with } f: I \longrightarrow\left\{R_{i} \mid i \in I\right\} .
$$

13. Halvorson adds, incorrectly as argued above, that such a syntactic view would be "indistinguishable from the oft-reviled 'received view of theories". 
Thus the family of an indexed structure is an interpretation with $I=\mathscr{V}$, since then $\left\langle A,\left\{R_{i}\right\}_{i \in I}\right\rangle=\langle A, \mathscr{J}\rangle$. Therefore an indexed relational structure is formally indistinguishable from a labelled relational structure.

More generally, a labelled structure $\langle A, \mathscr{I}\rangle$ is a pair of a domain $A$ and a mapping $\mathscr{I}$ from symbols to functions, relations, and constants. An indexed structure, $\left\langle A,\left\{R_{i}\right\}_{i \in I},\left\{F_{j}\right\}_{j \in J},\left\{c_{k}\right\}_{k \in K}\right\rangle$ is a tuple of a domain $A$, a family of relations, a family of functions, and a family of constants. Assuming that the index sets $I, J$, and $K$ are disjoint, this means that the indexed structure is definitionally equivalent to the pair $\left\langle A,\left\{W_{l}\right\}_{l \in I \cup J \cup K}\right\rangle$, where $\left\{W_{l}\right\}_{l \in I \cup J \cup K}$ is a mapping from $I \cup J \cup K$ to the set $\left\{W_{l} \mid l \in I \cup J \cup K\right\}:=\left\{R_{i} \mid i \in I\right\} \cup\left\{F_{j} \mid j \in J\right\} \cup\left\{c_{k} \mid k \in K\right\} .{ }^{14}$ Even if $I, J$, and $K$ are not disjoint, the indexed structure is definitionally equivalent to $\left\langle A,\left\{W_{l}\right\}_{l \in I^{*} \cup J^{*} \cup K^{*}}\right\rangle$, where $I^{*}:=\{\langle i, \varnothing, \varnothing\rangle \mid i \in I\}, J^{*}:=\{\langle\varnothing, j, \varnothing\rangle \mid j \in J\}$, and $K^{*}:=\{\langle\varnothing, \varnothing, k\rangle \mid k \in K\}$. In short, the families induce a mapping from their index sets to the relations, functions, and constants. And this mapping is indistinguishable from an interpretation, which is also a mapping from a set of symbols to the relations, functions, and constants.

In effect, the difference between an indexed structure and a labelled structure is that an indexed structure's index sets are not meant to be symbols of predicate logic. Of course, that does not hinder anyone to use them as such, and it also leaves nothing of the claim that when theories are given by indexed structures, there is some greater language independence. For two indexed structures are isomorphic given their index sets, so that changing the index sets of two isomorphic structures can render them non-isomorphic, and changing the index sets of two non-isomorphic structures and can sometimes render them isomorphic. A dependence on a vocabulary is thus exchanged for a dependence on an index set (which could be taken to be a vocabulary). Therefore there is no substantial middle ground between $\mathrm{H}$-structures (which are, as Halvorson shows, not expressive enough) and labelled structures. ${ }^{15}$

\section{Van Fraassen's Defense of Semantic Approaches}

Van Fraassen (2014) makes three "gentle remarks" about the discussion between Glymour and Halvorson:

1. The alleged dependence of any plausible semantic view on language is the trivial one already identified by himself (279),

2. Halvorson misrepresents (a) syntactic approaches and (b) semantic approaches (280-82), and

14. To be precise, and understanding families as functions, $\left.\left\{W_{l}\right\}_{l \in I \cup J \cup K}\right|_{I}=\left\{R_{i}\right\}_{i \in I}$, $\left.\left\{W_{l}\right\}_{l \in I \cup J \cup K}\right|_{j}=\left\{F_{j}\right\}_{j \in J}$, and $\left.\left\{W_{l}\right\}_{l \in I \cup J \cup K}\right|_{K}=\left\{c_{k}\right\}_{k \in K}$.

15. Another suggested middle ground that does not fall prey to Halvorson's argument equally turns out to be equivalent to labelled structures (Lutz 2014d, 483-84). 
3. Halvorson's arguments do not affect semantic approaches because they ignore the role of representation (van Fraassen 2014, \$2).

Van Fraassen's first remark starts from Glymour's claim that "Newton, Einstein, Schrodinger, and so on, had no way of specifying the class of relational structures they intended, except indirectly, as those structures satisfying their theoretical claims. Nor do we today" (Glymour 2013, 288). And these theoretical claims are given in a language. "Equations are sentences or formulas in a language", for instance (288-89). With a reference to his "trivial point" that "to present a theory, we must present it in and by language", van Fraassen $(2014, \$ 3$ ) responds that

there must be a serious point to Glymour's iteration of this trivial point. Either he wants to strongly endorse Halvorson's take on what the semantic view really is, or else he spends those several impassioned paragraphs to warn Halvorson away from tilting at windmills. I think of Professor Glymour as a person of serious intent, so the latter seems more likely.

Thus Glymour either makes van Fraassen's point clear to Halvorson, or he is not being serious. A third option is that Glymour and Halvorson agree on the role of language in semantic approaches and make a non-trivial point different from van Fraassen's. This more charitable interpretation of their exchange is not quite as obvious because Glymour $(2013,287)$ begins his discussion noting that a Suppes predicate is a predicate and thus is linguistic, which does suggest van Fraassen's trivial point. But Glymour's discussion of the relation between the two structures $\left\langle D_{1} ; \varphi, \chi, \varphi\right\rangle$ and $\left\langle D_{2} ; \tau, \delta, \omega\right\rangle$ leads him to the stronger conclusion that the structures involved in semantic approaches must be labelled ones. And this means that even if the Suppes predicate can be described by a multitude of languages different from predicate logic, the structure that it describes must determine a unique signature for predicate logic. Thus if van Fraassen $(1989,366)$ indeed thinks that the "impact of Suppes's innovation is lost if models are defined $[\ldots]$ to be partially linguistic entities, each yoked to a particular syntax", then Glymour argues that the impact of Suppes's innovation is lost. Recall that van Fraassen (1989, 212-13) claimed that "we must advance the conception of natural language as not being constituted by any one realization of any such logical skeleton [of predicate logic]. [...] In my opinion, it would be a poor choice to try and describe a whole language in which a given theory can be formulated." But if every structure is labelled, then each description of a theory contains the complete signature for a predicate logical realization in which the theory can be formulated. Hence there is much more than "a bow in the direction of syntactic description" in van Fraassen's semantic approach

Based on Toulmin's description of the Received View's ambition at its most grandiose, remark 2(a) relies on the assumption that syntactic approaches assume a mathesis universalis. Van Fraassen does not spell out Halvorson's misrepresentation, but it is presumably his assumption that syntactic approaches can be ap- 
plied to single theories. As noted above, this is not a misrepresentation, neither for syntactic approaches nor for the Received View.

For remark 2(b), van Fraassen $\left(2014, \mathbb{S}_{3}\right)$ refers to his earlier account of elementary sentences and satisfaction functions to show that he did not intend to avoid language altogether ("a trivially absurd idea") and that Halvorson therefore misrepresents van Fraassen's semantic account. But since van Fraassen (1989, 365, n. 34) "soon found it much more advantageous to concentrate on the propositions expressible by elementary statements, rather than on the statements themselves", this historical remark does not touch Halvorson's criticism, which applies to van Fraassen's current position with indexed structures and without elementary sentences. Of course, to make van Fraassen's trivial point, a reference to elementary sentences is not necessary (since even Suppes predicates must be phrased in some language). But neither the trivial point nor the use of elementary sentences addresses Halvorson's (and Glymour's) conclusion, which is that semantic approaches require the language of predicate logic and hence cannot rely only on natural language or elementary sentences alone.

Van Fraassen's remark 3 starts from an analogy: Treating a theory as a class of structures is like treating the Mona Lisa as a piece of wood. A more direct formulation is as follows: Semantic approaches assume that every theory can be described by an equivalence class of classes of structures; they do not assume that every such equivalence class describes a theory, for only those equivalence classes that represent do. This point is well taken, but it does not obviously invalidate Halvorson's criticisms if the representational aspect of the theories (the "theoretical hypothesis") is not further specified. The question is then whether van Fraassen's account of the theoretical hypothesis, empirical adequacy, escapes Halvorson's criticism.

Oddly, van Fraassen does not respond to Glymour's argument to the contrary. Glymour $(2013,289-90)$ points out that empirical adequacy is defined via embeddability, which is preserved under isomorphism. So a purely formal discussion like Halvorson's should be enough. More explicitly, one can directly address van Fraassen's criticism that "in the cases Halvorson discusses [...] it is not clear what the theoretical hypothesis is supposed to be. Halvorson does not address the problem of defining empirical adequacy" (van Fraassen 2014, n. 2). The problem can be addressed easily by supplying theoretical hypotheses. In van Fraassen's case, this means identifying specific substructures as empirical substructures. Their isomorphism with the appearances then establishes empirical adequacy. For the group example, the models of the theory formalized with ' $\circ$ ' and ' $e$ ' include structure $\langle\mathbb{R},+, 0\rangle$. Thus assume that the empirical substructure for this specific model is given by $\langle\mathbb{N},+, 0\rangle$. When formalized with 'o' alone, the models of the theory do not contain $\langle\mathbb{R},+, 0\rangle$ with empirical substructure $\langle\mathbb{N},+, 0\rangle$, but rather $\langle\mathbb{R},+\rangle$ with empirical substructure $\langle\mathbb{N},+\rangle$. Therefore the two classes of models of definitionally equivalent axiomatizations of the same theory are not empirically adequate for the same appearances, and are therefore incorrectly treated as describing different theories by van Fraassen's 
semantic approach. The models of Halvorson's theories $T=\{\exists x \forall y(x=y)\}$ and $T^{\prime}=T \cup\left\{\forall x\left(P_{1} x \rightarrow P_{2} x\right)\right\}$ each have only one substructure, namely themselves. Therefore the models are their own empirical substructures and $T$ and $T^{\prime}$ are theories only about observable phenomena. Since the models of $T$ and $T^{\prime}$ are pointwise $\mathrm{H}$-isomorphic, $T$ has a model that is $\mathrm{H}$-isomorphic to an appearance if and only if $T^{\prime}$ has such a model, and thus the classes of the models of the two theories are empirically adequate for the same appearances. Thus two different theories are incorrectly treated as the same. Therefore taking the representational aspect of theories into account does not avoid Halvorson's criticism. This also means that van Fraassen cannot rely on H-structures, but must rely on indexed structures, which, I have argued, amount to labelled structures.

\section{Not With a Bang, But a Whimper}

Halvorson's arguments have shown that plausible semantic approaches cannot rely on a notion of structure that consists of a domain and a multiset, but must rely on the notion of an indexed structure. The analysis of the notion of an indexed structure then shows that the alleged language independence of semantic approaches boils down to a decision not to call a specific mapping an interpretation, but a family. This makes no formal difference, and informally it is at best something of an indicator where one's interests lie: In the set theoretic aspects of the formalism or in the predicate logical aspects. Accordingly, there have been philosophers of science working on both aspects (e. g., Kemeny 1956; Przełęcki 1969; Demopoulos 2008; Andreas 2010; van Benthem 2012) without therefore being incoherent.

In spite of these results, a lot of effort has gone into disparaging semantic and especially syntactic approaches. Even van Fraassen (2014, 282), who wants to end his remarks "on a positive note" with the suggestion to let "a hundred flowers blossom" as far as the formalization of theories is concerned, cannot refrain from a Parthian shot at "the poverty-stricken syntactic approach of Carnap and his cohort". But van Fraassen's own account of empirical adequacy can be described in the Received View (Lutz 2014a), which is thus at least not more poverty-stricken than van Fraassen's account. Similarly, the partial structure approach, which has been presented as a generalization of the semantic approach based on model theory (da Costa and French 1990) and as decisively superior to the Received View (French and Ladyman 1999) can be described in the Received View as well (Lutz, forthcoming). So in spite of over 40 years of infighting in the philosophy of science, the syntax-semantics debate was about a distinction that marks no difference. 


\section{A Theory Independence of Embeddings}

Halvorson's argument discussed in $\$ 5.1$ is meant to establish that semantic approaches have no adequate notion of isomorphism because the only possible notion of isomorphism that fits semantic approaches fails (cf. Halvorson 2013, 476). Since an isomorphism can be defined as a surjective embedding, one could thus conclude that the semantic approach cannot explicate the relation of embedding between theories either. Halvorson $(2012,199)$ also argues directly for this latter claim. He states that, while the term 'embeddability' "has a technical use in model theory", "the semantic approach cannot explicate the relation of embedding between theories". This claim has some prima facie plausibility, given that van Fraassen himself seems to be working with a definition of isomorphism other than that of model theory: He switched from elementary statements and satisfaction functions to embeddings while retaining his commitment to formalizations in state-spaces (van Fraassen 1980, \$3.9; van Fraassen 1989, \$9.3,\$9.4), without however spelling out how embeddings work in state-spaces. If there is indeed no semantic account of embedding and since an embedding can be defined as an isomorphism to a substructure, Halvorson can conclude that the semantic approach cannot explicate the relation of isomorphism between theories either (Halvorson 2012, 201). ${ }^{16}$

Assuming that Halvorson's direct argument against the semantic approach's ability to explicate embedding succeeds, it provides a reason for van Fraassen to rely on the model theoretic notion of embedding. And in spite of initial appearances, van Fraassen must mean the model theoretic conceptions of isomorphism and embedding when using these terms in his definition of empirical adequacy (Lutz 2014b, 3206-8), the most obvious being his reference to model theoretic structures as the meaning of 'model' discussed above (van Fraassen 2014, 277).

Glymour and I have argued that the notions of isomorphism and embedding defined in model theory (whether for indexed or labelled structures) are appropriate for semantic approaches. Halvorson $(2012,199)$ to the contrary claims that model theoretic embeddability describes "a relation between models of a single theory", which would render both embeddings and isomorphisms incapable of relating different theories. But this claim is false: The models of one theory (say, ' $\exists x \forall y(x=y)$ ') may be embeddable in the models of an incompatible theory (say, ' $\exists x y[x \neq y \wedge \forall z(z=x \vee z=y)]$ '), and two non-equivalent theories (say, ' $\exists x P x$ ' and ' $\forall x P x$ ') can share a model and thus have isomorphic models. More generally, neither the definition of embedding nor that of isomorphism references theories. At the most, embeddings and isomorphisms describe relations between structures of a single signature or index set (cf. Hodges 1993, 1, 5).

16. This argument assumes that even outside of model theory, embeddings should be defined as isomorphisms to substructures, whatever these terms mean. 


\section{References}

Andreas, Holger. 2010. "Semantic Holism in Scientific Language." Philosophy of Science 77 (4): 524-543. doi:10.1086/656007.

Andréka, Hajnal, Judit X. Madarász, and István Németi. 2005. "Mutual Definability Does Not Imply Definitional Equivalence, a Simple Example.” Mathematical Logic Quarterly 51 (6): 591-597. doi:10.1002/malq.200410051.

Andrews, Peter B. 2002. An Introduction to Mathematical Logic and Type Theory: To Truth Through Proof. 2nd ed. Vol. 27. Applied Logic Series. Dordrecht: Kluwer Academic Publishers.

Bell, John L., and Alan B. Slomson. 1974. Models and Ultraproducts: An Introduction. 3rd ed. Amsterdam: North-Holland.

Blizard, Wayne D. 1989. "Multiset Theory." Notre Dame Journal of Formal Logic 30 (1): 36-66. doi:10.1305/ndjfl/1093634995.

Bourbaki, Nicolas. 1968. Theory of Sets. Elements of Mathematics. Paris: Hermann.

Carnap, Rudolf. 1936. "Wahrheit und Bewährung." In Actes du Congrès international de philosophie scientifique, Sorbonne, Paris 1935, 4: Induction et probabilité, 391:18-23. Actualités scientifiques et industrielles. Paris: Hermann \& $\mathrm{C}^{\mathrm{ie}}$.

1939. Foundations of Logic and Mathematics. I,3:139-213. Foundations of the Unity of Science. Toward an International Encyclopedia of Unified Science. References are to the two-volume edition. Chicago and London: University of Chicago Press.

1956. "The Methodological Character of Theoretical Concepts." In The Foundations of Science and the Concepts of Psychology and Psychoanalysis, edited by Herbert Feigl and Michael Scriven, 1:38-75. Minnesota Studies in the Philosophy of Science. Minneapolis, MN: University of Minnesota Press.

Chang, Chen Chung, and H. Jerome Keisler. 1990. Model Theory. 3rd ed. Vol. 73. Studies in Logic and the Foundations of Mathematics. 3rd impression 1992. Amsterdam: North Holland.

Chang, Hasok. 2005. "A Case for Old-fashioned Observability, and a Reconstructed Constructive Empiricism." Philosophy of Science 72 (5): 876-887. doi:10.1086/508116.

Da Costa, Newton C. A., and Steven French. 1990. "The Model-Theoretic Approach in the Philosophy of Science." Philosophy of Science 57 (2): 248-65. 
Demopoulos, William. 2008. "Some Remarks on the Bearing of Model Theory on the Theory of Theories." Synthese 164 (3): 359-383. doi:10.1007/s11229o०8-9355-1.

Feigl, Herbert. 1970. "The "Orthodox" View of Theories: Remarks in Defense as well as Critique." In Analyses of Theories and Methods of Physics and Psychology, edited by Michael Radner and Stephen Winokur, 4:3-16. Minnesota Studies in the Philosophy of Science. Minneapolis, MN: University of Minnesota Press.

French, Steven, and James Ladyman. 1999. "Reinflating the Semantic Approach." International Studies in the Philosophy of Science 13 (2): 103-121. doi:10.1080/ 02698599908573612.

Frigg, Roman, and Stephan Hartmann. 2008. "Models in Science." In The Stanford Encyclopedia of Philosophy, Spring 2008, edited by Edward N. Zalta. Stanford: The Metaphysics Research Lab, Center for the Study of Language and Information, Stanford University.

Glymour, Clark. 2013. "Theoretical Equivalence and the Semantic View of Theories." Philosophy of Science 80 (2): 286-297. doi:10.1086/670261.

Halvorson, Hans. 2012. "What Scientific Theories Could Not Be." Philosophy of Sciences 79 (2): 183-206. doi:10.1086/664745.

- 2013. "The Semantic View, if Plausible, is Syntactic." Philosophy of Science 80 (3): 475-478. doi:10.1086/671077.

Hein, James L. 2003. Discrete Mathematics. 2nd ed. Sudbury, MA: Jones \& Bartlett Publishers.

Hendry, R. F., and Stathis Psillos. 2007. "How to do Things with Theories: An Interactive View of Language and Models in Science." In The Courage of Doing Philosophy: Essays Dedicated to Leszek Nowak, edited by Jerzy Brzeziński, Andrzej Klawiter, Theo A.F. Kuipers, Krzysztof Łastowski, Katarzyna Paprzycka, and Piotr Przybysz, 59-115. Amsterdam/New York: Rodopi.

Hodges, Wilfrid. 1993. Model Theory. Vol. 42. Encyclopedia of Mathematics and its Applications. Digitally printed in 2008. Cambridge: Cambridge University Press.

Kemeny, John G. 1956. “A New Approach to Semantics-Part I.” The Journal of Symbolic Logic 21 (1): 1-27.

Leivant, Daniel. 1994. "Higher Order Logic.” In Deduction Methodologies, edited by Dov M. Gabbay, C. J. Hogger, and J. A. Robinson, 2:229-321. Handbook of Logic in Artificial Intelligence and Logic Programming. Oxford: Oxford University Press. 
Lutz, Sebastian. 2012. "On a Straw Man in the Philosophy of Science: A Defense of the Received View." HOPOS: The Journal of the International Society for the History of Philosophy of Science 2 (1): 77-120. doi:10.1086/664460.

- 2014a. "Empirical Adequacy in the Received View." Philosophy of Science 81 (5): 1171-1183. doi:10.1086/677886.

- 2014b. "Generalizing Empirical Adequacy I: Multiplicity and Approximation." Synthese 191:3195-3225. doi:10.1007/s11229-014-0440-3.

- 2014c. "The Semantics of Scientific Theories." In Ksiegga pamiatkowa Marianowi Przetęckiemu w darze na 9o-lecie urodzin, edited by Anna Brożek and Jacek Jadacki, 33-67. Authoritative preprint: http:// philsci-archive.pitt. edu/id/eprint/9630. Lublin: Nobertinum.

. 2014d. "What's Right With a Syntactic Approach to Theories and Models?” Erkenntnis 79 (8 Supplement): 1475-1492. doi:10.1007/s10670-013-95785 .

—. Forthcoming. "Partial Model Theory as Model Theory." Ergo.

Muller, F. A. 2010. "Reflections on the Revolution at Stanford." Synthese 183 (1): 87-114. doi:10.1007/s11229-009-9669-7.

Newman, M. H. A. 1928. "Mr. Russell's “Causal Theory of Perception”.” Mind 37 (146): $137-148$.

Przełęcki, Marian. 1969. The Logic of Empirical Theories. Monographs in Modern Logic Series. London/New York: Routledge \& Kegan Paul/Humanities Press.

Putnam, Hilary. 1962. "What Theories Are Not." In Logic, Methodology, and Philosophy of Science: Proceedings of the 1960 International Congress, edited by Ernest Nagel, Patrick Suppes, and Alfred Tarski, 240-251. Stanford: Stanford University Press.

1989. "Model Theory and the "Factuality" of Semantics." In Reflections on Chomsky, edited by Alex George, 213-232. References are to the reprint (Putnam 1994, ch. 18). Oxford: Basil Blackwell.

- 1994. Words and Life. Second printing 1995, edited by James Conant. Cambridge, MA: Harvard University Press.

Robinson, Abraham. 1963. Introduction to Model Theory and to the Metamathematics of Algebra. Amsterdam: North-Holland Publishing Company.

Schurz, Gerhard. 2014. Philosophy of Science. A Unified Approach. New York, NY, and London: Routledge.

Suárez, Mauricio, and Nancy Cartwright. 2008. "Theories: Tools versus models." Studies in History and Philosophy of Modern Physics 39:62-81. 
Suppe, Frederick. 1971. “On Partial Interpretation.” The Journal of Philosophy 68 (3): 57-76. doi:10.2307/2025168.

. 1972. "What's Wrong with the Received View on the Structure of Scientific Theories?" Philosophy of Science 39 (1): 1-19.

- 1974a. "The Search for Philosophic Understanding of Scientific Theories." In Suppe 1974b, 3-241.

ed. 1974b. The Structure of Scientific Theories. Urbana, IL: University of Illinois Press.

- 2000. "Understanding Scientific Theories: An Assessment of Developments, 1969-1998." Supplement. Proceedings of the 1998 Biennial Meetings of the Philosophy of Science Association. Part II: Symposia Papers, Philosophy of Science 67:S102-S115.

Suppes, Patrick. 1957. Introduction to Logic. New York: Van Nostrand Reinhold Company.

1960. "A Comparison of the Meaning and Uses of Models in Mathematics and the Empirical Sciences." Synthese 12:287-301.

1967. "What is a Scientific Theory?" In Philosophy of Science Today, edited by Sidney Morgenbesser, 55-67. New York: Basic Books.

- 2002. Representation and Invariance of Scientific Structures. Stanford, CA: CSLI Publications.

Tarski, Alfred. 1935. "Einige methodologische Untersuchungen über die Definierbarkeit der Begriffe.” Erkenntnis 5 (1): 80-100.

- 1944. "The Semantic Conception of Truth and the Foundations of Semantics." Philosophy and Phenomenological Research 4 (3): 341-376. doi:10. $2307 / 2102968$.

Thompson, Paul. 2007. "Formalisations of Evolutionary Biology." In Philosophy of Biology, edited by Mohan Matthen and Christopher Stephens, 3:485-523. Handbook of the Philosophy of Science. Amsterdam: Elsevier.

Toulmin, Stephen. 1974. “The Structure of Scientific Theories.” In Suppe 1974b, 600-614.

Turney, Peter. 1990. "Embeddability, Syntax, and Semantics in Accounts of Scientific Theories." Journal of Philosophical Logic 19:429-451. doi:10.1007/ BFoo263319.

Väänänen, Jouku. 2001. "Second-Order Logic and Foundations of Mathematics." The Bulletin of Symbolic Logic 7 (4): 504-520. 
Van Benthem, Johan. 2012. "The Logic of Empirical Theories Revisited." Synthese 186 (3): 775-792. doi:10.1007/s11229-011-9916-6.

Van Fraassen, Bas C. 1970. "On the Extension of Beth's Semantics of Physical Theories." Philosophy of Science 37 (3): 325-339.

1980. The Scientific Image. The Clarendon Library of Logic and Philosophy. Oxford: Clarendon Press.

. 1987. "The Semantic Approach to Scientific Theories." In The Process of Science. Contemporary Philosophical Approaches to Understanding Scientific Practice, edited by Nancy J. Nersessian, 105-124. Science and Philosophy. Dordrecht, The Netherlands: Martinus Nijhoff Publishers.

1989. Laws and Symmetry. The Clarendon Library of Logic and Philosophy. Oxford: Clarendon Press.

- 2002. The Empirical Stance. The Terry Lectures. New Haven, CT: Yale University Press.

2008. Scientific Representation: Paradoxes of Perspective. Oxford: Clarendon Press.

- 2014. "One or Two Gentle Remarks About Hans Halvorson's Critique of the Semantic View." Philosophy of Science 81 (2): 276-283. doi:10.1086/ 675645 .

Whitehead, Alfred North, and Bertrand Russell. 1927/1929. Principia Mathematica. 2nd ed. Three volumes. Cambridge: Cambridge University Press.

Woodger, Joseph H. 1939. The Technique of Theory Construction. Vol. II,5. Foundations of the Unity of Science. Toward an International Encyclopedia of Unified Science. References are to the two-volume edition. Chicago and London: University of Chicago Press.

Worrall, John. 1984. "An Unreal Image." Review of (van Fraassen 1980), The British Journal for the Philosophy of Science 35:65-80. 